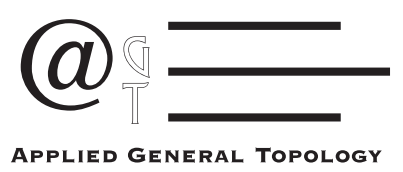

Applied General Topology

(C) Universidad Politécnica de Valencia

Volume 10, No. 1, 2009

pp. $69-83$

\title{
F-supercontinuous functions
}

\author{
J. K. Kohli, D. Singh* and Jeetendra Aggarwal
}

\begin{abstract}
A strong variant of continuity called ' $F$-supercontinuity' is introduced. The class of $F$-supercontinuous functions strictly contains the class of $z$-supercontinuous functions (Indian J. Pure Appl. Math. 33 (7) (2002), 1097-1108) which in turn properly contains the class of cl-supercontinuous functions ( $\equiv$ clopen maps) (Appl. Gen. Topology 8 (2) (2007), 293-300; Indian J. Pure Appl. Math. 14 (6) (1983), 762-772). Further, the class of $F$-supercontinuous functions is properly contained in the class of $R$-supercontinuous functions which in turn is strictly contained in the class of continuous functions. Basic properties of $F$-supercontinuous functions are studied and their place in the hierarchy of strong variants of continuity, which already exist in the mathematical literature, is elaborated. If either domain or range is a functionally regular space (Indagationes Math. 15 (1951), 359-368; 38 (1976), 281-288), then the notions of continuity, F-supercontinuity and R-supercontinuity coincide.
\end{abstract}

2000 AMS Classification: 54C08, 54C10, 54D10, 54D20, 54D30

Keywords: z-supercontinuous function, F-supercontinuous function, functionally regular space, functionally Hausdorff space, F-completely regular space, F-quotient topology

\section{INTRODUCTION}

Several strong variants of continuity occur in the lore of mathematical literature which arise in many branches of mathematics and applications of mathematics.

In many situations in topology, analysis and other disciplines continuity is not sufficient and a strong form of continuity is required to meet the demand of a particular situation. The strong variants of continuity with which we shall be dealing in this paper include, among others, are strongly continuous functions [16], perfectly continuous functions [20], clopen maps [21] (三 cl-supercontinuous

*This research was partially supported by University Grants Commission, India. 
functions [23]), $z$-supercontinuous functions [8], $D$-supercontinuous functions [10], $D^{*}$-supercontinuous functions [22], $D_{\delta}$-supercontinuous functions [11], strongly $\theta$-continuous functions [19] and supercontinuous functions [18]. The main purpose of this paper is to introduce a new class of functions called ' $F$ supercontinuous functions', study their basic properties and discuss their place in the hierarchy of strong variants of continuity that already exist in the mathematical literature. The notion of $F$-supercontinuous functions arise naturally in case either domain or range is a functionally regular space ([1], [2]). It turns out that the class of $F$-supercontinuous functions properly includes the class of $z$-supercontinuous functions [8] and is strictly contained in the class of $R$ supercontinuous functions [14] which in turn is properly contained in the class of continuous functions. Further if either domain or range is a functionally regular space ([1], [2]) then all the three classes of (i) $F$-supercontinuous functions (ii) $R$-supercontinuous functions, and (iii) continuous functions coincide. Moreover, if either $X$ or $Y$ is a completely regular space, then all these three classes of functions are identical with the class of $z$-supercontinuous functions [8]. Furthermore, if either domain or range is zero dimensional space, then all the four above classes of functions coincide with the class of cl-supercontinuous functions ([21], [23]).

Section 2 is devoted to the preliminaries and basic definitions. In Section 3, we introduce the notion of ' $F$-supercontinuous function' and elaborate on its place in the hierarchy of strong variants of continuity which already exist in the literature. Basic properties of $F$-supercontinuous functions are studied in Section 4, while properties of graph of an $F$-supercontinuous function are discussed in Section 5. Interplay of topological properties and F-supercontinuous functions is investigated in Section 6 and the notion of $F$-quotient topology is formulated in Section 7. Change of topology of a topological space $(X, \tau)$ into a functionally regular topology $\tau_{F}$ and a completely regular topology $\tau_{z}$ are considered in Section 8 wherein interrelations between $\tau, \tau_{F}$ and $\tau_{z}$ are elaborated and alternative proofs of certain results in the preceding sections are suggested.

\section{BASIC DEFINITIONS AND PRELIMINARIES}

A collection $\beta$ of subsets of a space $X$ is called as open complementary system [6] if $\beta$ consists of open sets such that for every $B \in \beta$, there exist $B_{1}, B_{2}, \ldots \in \beta$ with $B=\cup\left\{X \backslash B_{i}: i \in N\right\}$. A subset $A$ of a space $X$ is called a strongly open $F_{\sigma}$-set $[6]$ if there exists a countable open complementary system $\beta(A)$ with $A \in \beta(A)$. The complement of a strongly open $F_{\sigma}$-set is called strongly closed $G_{\delta}$-set. A subset $A$ of a space $X$ is called a regular $G_{\delta}$-set [17] if A is an intersection of a sequence of closed sets whose interiors contain $A$, i.e., if $A=\bigcap_{n=1}^{\infty} F_{n}=\bigcap_{n=1}^{\infty} F_{n}^{\circ}$, where each $F_{n}$ is a closed subset of $X$. The complement of a regular $G_{\delta}$-set is called a regular $F_{\sigma}$-set. An open subset $A$ of a space $X$ is said to be $r$-open [14] if it is expressible as a union of closed sets. 
Definition 2.1. A function $f: X \rightarrow Y$ from a topological space $X$ into a topological space $Y$ is said to be

(a) strongly continuous [16] if $f(\bar{A}) \subset f(A)$ for each subset $A$ of $X$.

(b) perfectly continuous [20] if $f^{-1}(V)$ is clopen in $X$ for every open set $V \subset Y$.

(c) cl-supercontinuous [23] (三 clopen map [21]) if for each $x \in X$ and each open set $V$ containing $f(x)$ there is a clopen set $U$ containing $x$ such that $f(U) \subset V$.

(d) z-supercontinuous [8] if for each $x \in X$ and for each open set $V$ containing $f(x)$, there exists a cozero set $U$ containing $x$ such that $f(U) \subset V$.

(e) $D_{\delta}$-supercontinuous [11] if for each $x \in X$ and for each open set $V$ containing $f(x)$, there exists a regular $F_{\sigma}$ set $U$ containing $x$ such that $f(U) \subset V$.

(f) D-supercontinuous [10] if for each $x \in X$ and each open set $U$ containing $f(x)$ there exists an open $F_{\sigma}$-set $V$ containing $x$ such that $f(V) \subset U$.

(g) $D^{*}$-supercontinuous [22] if for each $x \in X$ and each open set $U$ containing $f(x)$ there exists a strongly open $F_{\sigma}$-set $V$ containing $x$ such that $f(V) \subset U$.

(h) strongly $\theta$-continuous [19] if for each $x \in X$ and for each open set $V$ containing $f(x)$, there exists an open set $U$ containing $x$ such that $f(\bar{U}) \subset$ $V$.

(i) R-supercontinuous [14] if for each $x \in X$ and each open set $U$ containing $f(x)$ there exists an $r$-open set $V$ containing $x$ such that $f(V) \subset U$.

(j) supercontinuous [18] if for each $x \in X$ and for each open set $V$ containing $f(x)$, there exists a regular open set $U$ containing $x$ such that $f(U) \subset V$.

Definition 2.2. A topological space $X$ is said to be

(i) functionally regular ([1], [2]) if for each closed set $A$ and a point $x \notin A$ there exists a continuous real-valued function $f$ defined on $X$ such that $f(x) \notin f(A)$; or equivalently for each $x \in X$ and each open set $U$ containing $x$ there exists a zero set $Z$ such that $x \in Z \subset U$.

(ii) D-regular space [6] if it has a base of open $F_{\sigma}$-sets.

(iii) functionally Hausdorff [25] If for $x, y \in X, x \neq y$ there exists a continuous function $f: X \rightarrow[0,1]$ such that $f(x) \neq f(y)$.

(iv) countably $H$-closed if it is Hausdorff and every countable open cover of $X$ has a finite subcollection whose union is dense in $X$.

(v) semiregular if it has a base of regular open sets.

In order to systematize the study of separation by continuous real-valued functions, Van Est and Freudenthal [25] introduced the notion of a functionally regular space and showed its distinctiveness from the standard separation axioms and other separation axioms defined by them. Further properties of functionally regular spaces have been studied by Aull ([1], [2]). 


\section{3. $F$-SUPERCONTINUOUS FUnCtions}

An open set $U$ in a space $X$ is said to be $F$-open if for each $x \in U$, there exists a zero set $Z$ in $X$ such that $x \in Z \subset U$, or equivalently, $U$ is expressible as a union of zero sets. The complement of an $F$-open set will be referred to as an $F$-closed set.

Definition 3.1. A function $f: X \rightarrow Y$ from a topological space $X$ into a topological space $Y$ is said to be F-supercontinuous if for each $x \in X$ and each open set $U$ containing $f(x)$ there exists an $F$-open set $V$ containing $x$ such that $f(V) \subset U$.

The following diagram reflects upon the place of $F$-supercontinuous functions in the hierarchy of strong variants of continuity that already exist in the literature. The implications are either well known or immediately follow from definitions.

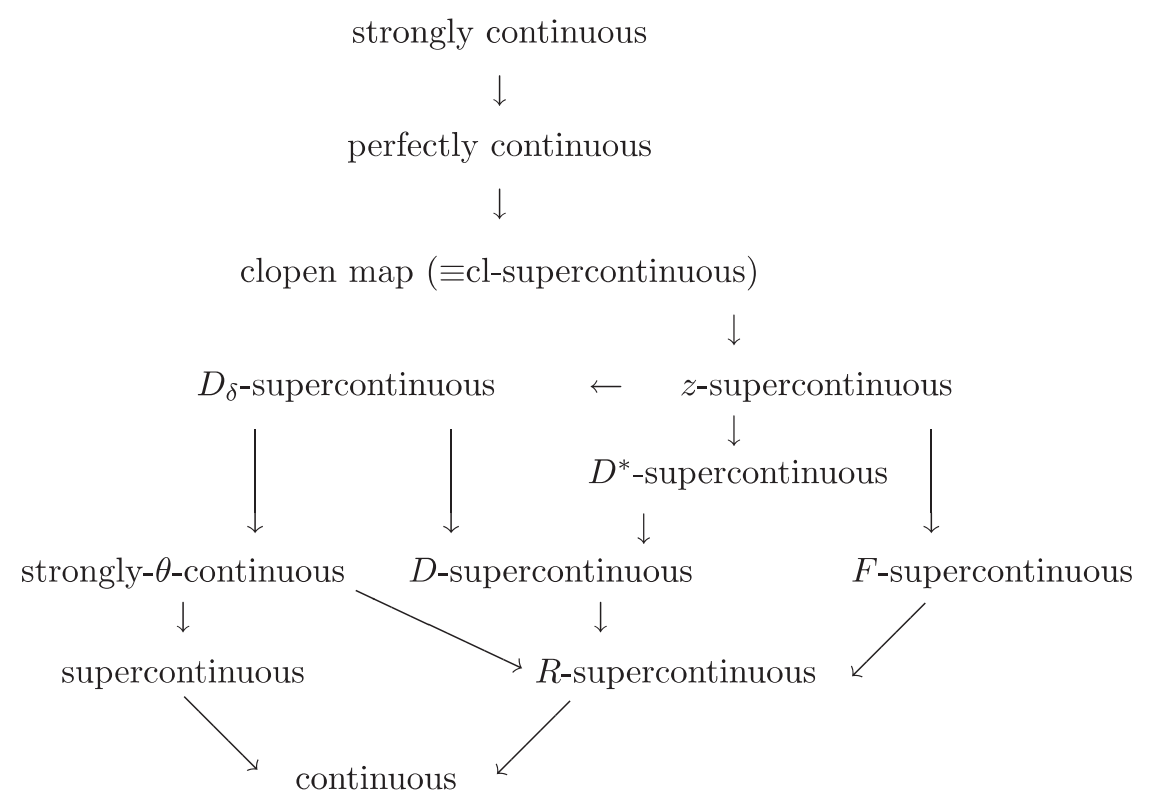

However, none of the above implications is reversible which is either well known or follows from the following observations and examples.

\section{Observations and Examples}

3.2 If either $X$ or $Y$ is a functionally regular space, then every continuous function $f: X \rightarrow Y$ is $F$-supercontinuous and hence $R$-supercontinuous.

3.3 If either $X$ or $Y$ is a completely regular space, then every continuous function $f: X \rightarrow Y$ is $z$-supercontinuous. 
3.4 Let $X=Y$ be the regular space due to Hewitt [7] on which every continuous real valued function is constant and let $f$ denote the identity map defined on $X$. Then $f$ is strongly $\theta$-continuous and so $R$-supercontinuous but it is not $F$-supercontinuous.

3.5 Let $X$ be a functionally regular space which is not completely regular and let $Y=X$. Then the identity mapping defined on $X$ is $F$-supercontinuous but not $z$-supercontinuous.

3.6 Let $X$ be the space of [3, Exercise 24, p. 139]. Then $X$ is a Hausdorff semiregular space which is not regular. Further, Aull pointed out that the space $X$ is a functionally regular space (see [1, Example 3]). Let $f$ denote the identity mapping defined on $X$. Then the function $f$ is supercontinuous as well as $F$-supercontinuous but not strongly $\theta$-continuous.

3.7 Let us denote by $X$ the space of Arens square [24, Example 80, p. 98]. Then $X$ is a Hausdorff space which is not functionally Hausdorff and hence not a functionally regular space. Also, $X$ is semiregular but not regular. So the identity mapping defined on $X$ is supercontinuous but not $F$-supercontinuous.

3.8 Let us denote by $X$ the space of irregular lattice topology [24, Example 79, p. 97]. Then $X$ is a functionally Hausdorff Lindelöf space which is not a semiregular space. In view of [1, Theorem 3], the space $X$ is a functionally regular space. Let $f$ denote the identity mapping defined on $X$. Then $f$ is an $F$-supercontinuous function but not supercontinuous.

3.9 Let us denote by $X$ the real line with the smallest topology generated by the Euclidean topology and the cocountable topology on $X$. The space $X$ is a functionally regular space, since it is a functionally Hausdorff Lindelöf space (see [1, Theorem 3]). The space $X$ is not $D$-regular, since $X$ is not a subparacompact space and every Lindelöf, $D$-regular space is subparacompact (see [4, Theorem 2]). Then the identity mapping defined on $X$ is an $F$-supercontinuous function but not a $D$-supercontinuous function.

Proposition 3.10. Let $f: X \rightarrow Y$ be a continuous function, defined on a functionally Hausdorff Lindelöf space $X$. Then $f$ is F-supercontinuous.

Proof. Since a functionally Hausdorff Lindelöf space is functionally regular (see [1]) and since every continuous function defined on a functionally regular space is $F$-supercontinuous, $f$ is $F$-supercontinuous.

Proposition 3.11. Let $f: X \rightarrow Y$ be a continuous function. If $X$ is a countably paracompact functionally regular space, then $f$ is $F$-supercontinuous as well as strongly $\theta$-continuous.

Proof. Since every continuous function defined on a functionally regular space is $F$-supercontinuous, so is $f$. Again, since every countably paracompact functionally regular space is regular (see [1]), and since every continuous function defined on a regular space is strongly $\theta$-continuous, $f$ is strongly $\theta$-continuous. 
Proposition 3.12. Let $f: X \rightarrow Y$ be a continuous function defined on a countably compact functionally regular space $X$. Then $f$ is z-supercontinuous.

Proof. This is immediate in view of the fact that every countably compact functionally regular space is completely regular (see [1]), and every continuous function defined on a completely regular space is $z$-supercontinuous.

Proposition 3.13. Let $f: X \rightarrow Y$ be a continuous function defined on $a$ countably $H$-closed, semiregular, functionally regular space $X$. Then $f$ is $z$ supercontinuous.

Proof. This is immediate from the fact that a countably $H$-closed, semiregular, functionally regular space $X$ is completely regular (see [1]).

\section{BAsic PRoperties of F-SUPERCONTINuOUs FunCtions}

Theorem 4.1. For a function $f: X \rightarrow Y$ from a topological space $X$ into a topological space $Y$, the following statements are equivalent:

(a) $f$ is F-supercontinuous.

(b) The inverse image of every open subset of $Y$ is $F$-open in $X$.

(c) The inverse image of every closed subset of $Y$ is F-closed in $X$.

(d) The inverse image of every subbasic open subset of $Y$ is F-open in $X$.

Proof. It is easy using definitions.

Definition 4.2. Let $X$ be a topological space and let $A \subset X$. A point $x \in X$ is said to be an $\mathbf{F}$-adherent point of the set $A$ if every $F$-open set containing $x$ has non-empty intersection with $A$. Let $A_{F}$ denote the set of all $F$-adherent points of the set $A$. The set $A$ is $F$-closed if and only if $A=A_{F}$.

Theorem 4.3. For a function $f: X \rightarrow Y$ the following statement are equivalent.

(a) $f$ is F-supercontinuous.

(b) $f\left(A_{F}\right) \subset \overline{f(A)}$ for every $A \subset X$.

(c) $\left(f^{-1}(B)\right)_{F} \subset f^{-1}(\bar{B})$ for every $B \subset Y$.

Proof. (a) $\Rightarrow$ (b). Since $\overline{f(A)}$ is closed in $Y$, by Theorem $4.1 f^{-1}(\overline{f(A)})$ is an $F$ closed set in $X$. Again, since $A \subset f^{-1}(\overline{f(A)}), A_{F} \subset\left[f^{-1}(\overline{f(A)})\right]_{F}=f^{-1}(\overline{f(A)})$ and so $f\left(A_{F}\right) \subset f\left(f^{-1}(\overline{f(A)})\right) \subset \overline{f(A)}$.

(b) $\Rightarrow$ (c). Let $B \subset Y$. Then $f\left(\left(f^{-1}(B)\right)_{F}\right) \subset \overline{f\left(f^{-1}(B)\right)} \subset \bar{B}$ and so it follows that $\left(f^{-1}(B)\right)_{F} \subset f^{-1}(\bar{B})$.

$(\mathrm{c}) \Rightarrow$ (a). Let $B$ be any closed set in $Y$. Then $\left(f^{-1}(B)\right)_{F} \subset f^{-1}(B)$. Since $f^{-1}(B) \subset \overline{f^{-1}(B)} \subset\left(f^{-1}(B)\right)_{F}, f^{-1}(B)=\left(f^{-1}(B)\right)_{F}$ which in turn implies that $f$ is $F$-supercontinuous.

Definition 4.4. A filterbase $\mathcal{F}$ is said to $\mathbf{F}$-converge to a point $x$, written as $\mathcal{F} \stackrel{F}{\rightarrow} x$ if every $F$-open set containing $x$ contains a member of $\mathcal{F}$.

Theorem 4.5. A function $f: X \rightarrow Y$ is F-supercontinuous if and only if for each $x \in X$ and each filter base $\mathcal{F}$ in $X$ that $F$-converges to $x, f(\mathcal{F}) \rightarrow f(x)$. 
Proof. Suppose that $f$ is $F$-supercontinuous and let $\mathcal{F}$ be a filter base in $X$ that $F$-converges to $x$. To show that the filter base $f(\mathcal{F})$ converges to $f(x)$, let $W$ be any open set containing $f(x)$. Then $x \in f^{-1}(W)$ and $f^{-1}(W)$ is $F$-open. Since the filter base $\mathcal{F}$ converge to $x$, there exists $F \in \mathcal{F}$ such that $F \subset f^{-1}(W)$. Then $f(F) \subset f\left(f^{-1}(W)\right) \subset W$ and so $f(\mathcal{F}) \rightarrow f(x)$.

Conversely, let $W$ be an open set containing $f(x)$. Now, the filter $\mathcal{F}$ generated by the filterbase $\mathcal{B}_{x}$ consisting of $F$-open sets containing $x, F$-converges to $x$. Since by hypothesis $f(\mathcal{F}) \rightarrow f(x)$, there exists a member $f(F)$ of $f(\mathcal{F})$ such that $f(F) \subset W$. Choose $B \in \mathcal{B}_{x}$ such that $B \subset F$. Since $B$ is an $F$-open set containing $x$ and since $f(B) \subset f(F) \subset W, f$ is $F$-supercontinuous.

Theorem 4.6. If $f: X \rightarrow Y$ is $F$-supercontinuous and $g: Y \rightarrow Z$ is continuous, then the composition $g \circ f$ is $F$-supercontinuous. In particular, the composition of F-supercontinuous functions is F-supercontinuous.

In general $F$-supercontinuity of $g \circ f$ need not imply even continuity of $f$. For example, let $X$ be the real line with cofinite topology, $Y$ be the real line with cocompact topology and $Z$ be the real line with indiscrete topology. Let $f: X \rightarrow Y$ and $g: Y \rightarrow Z$ be the identity mappings. Then $g \circ f$ and $g$ are $F$-supercontinuous. However, $f$ is not continuous.

It is routine to verify that $F$-supercontinuity is invariant under restrictions and enlargement of range.

Definition 4.7. A function $f: X \rightarrow Y$ is said to be $\mathbf{F}$-open (F-closed) if $f(A)$ is open (closed) in $Y$ for every $F$-open $(F$-closed) set $A$ in $X$.

Theorem 4.8. Let $f: X \rightarrow Y$ be an $F$-open ( $F$-closed), $F$-supercontinuous surjection and $g: Y \rightarrow Z$ be any function. Then the composition $g \circ f$ is $F$ supercontinuous if and only if $g$ is continuous. Further, if in addition $f$ maps $F$ open $(F$-closed) set to F-open $(F$-closed) set, then $g$ is an $F$-supercontinuous function.

Proof. Suppose that $g \circ f$ is $F$-supercontinuous. To show that $g$ is continuous, let $W$ be an open (closed) subset of $Z$. Then by Theorem $4.1(g \circ f)^{-1}(W)=$ $f^{-1}\left(g^{-1}(W)\right)$ is $F$-open ( $F$-closed) in $X$. Since $f$ is an $F$-open ( $F$-closed) surjection $f\left(f^{-1}\left(g^{-1}(W)\right)\right)=g^{-1}(W)$ is open (closed) in $Y$ and so $g$ is continuous.

Conversely, suppose that $g$ is continuous and let $W$ be an open (closed) set in $Z$. Then $g^{-1}(W)$ is open (closed) in $Y$. Since $f$ is $F$-supercontinuous, $f^{-1}\left(g^{-1}(W)\right)=(g \circ f)^{-1}(W)$ is $F$-open $(F$-closed $)$ in $X$ and so $g \circ f$ is $F$ supercontinuous.

For the last assertion we need only note that $F$-openness ( $F$-closedness) of $g^{-1}(W)$ ensures the $F$-supercontinuity of $g$.

Theorem 4.9. Let $f: X \rightarrow Y$ be any function. Then the following statements are true.

(a) If $\left\{U_{\alpha}: \alpha \in \Lambda\right\}$ is an F-open cover of $X$ and for each $\alpha, f_{\alpha}=f \mid U_{\alpha}$ is $F$-supercontinuous, then $f$ is $F$-supercontinuous. 
(b) If $\left\{F_{i}: i=1, \ldots, n\right\}$ is an $F$-closed cover of $X$ and $f_{i}=f \mid F_{i}$ is $F$ supercontinuous, then $f$ is $F$-supercontinuous.

Proof. (a) Let $V$ be any $F$-open set in $Y$. Then $f^{-1}(V)=\cup\left\{f_{\alpha}^{-1}(V): \alpha \in \Lambda\right\}$. Since each $f_{\alpha}$ is $F$-supercontinuous, In view of Theorem 4.1 each $f_{\alpha}^{-1}(V)$ is $F$ open in $U_{\alpha}$ and hence in $X$. Since any union of $F$-open sets is $F$-open, $f^{-1}(V)$ is $F$-open.

(b) Let $B$ be any $F$-closed set in $Y$. Then $f^{-1}(B)=\bigcup_{i=1}^{n} f_{i}^{-1}(B)$. Since each $f_{i}$ is $F$-supercontinuous, by Theorem 4.1 each $f_{i}^{-1}(B)$ is $F$-closed in $F_{i}$ and hence in $X$. Then $f^{-1}(B)$ being a finite union of $F$-closed sets is $F$-closed. So $f$ is $F$-supercontinuous.

Theorem 4.10. Let $\left\{f_{\alpha}: X \rightarrow X_{\alpha}: \alpha \in \Lambda\right\}$ be a family of functions and let $f: X \rightarrow \prod_{\alpha \in \Lambda} X_{\alpha}$ be defined by $f(x)=\left(f_{\alpha}(x)\right)$ for each $x \in X$. Then $f$ is $F$-supercontinuous if and only if each $f_{\alpha}: X \rightarrow X_{\alpha}$ is F-supercontinuous.

Proof. Let $f: X \rightarrow \prod_{\alpha \in \Lambda} X_{\alpha}$ be $F$-supercontinuous. Then the composition $p_{\alpha} \circ f=f_{\alpha}$, where $p_{\alpha}$ denotes the projection of $\prod_{\alpha \in \Lambda} X_{\alpha}$ onto $\alpha^{t h}$-coordinate space $X_{\alpha}$. So in view of Theorem 4.6 each $f_{\alpha}$ is $F$-supercontinuous.

Conversely, suppose that each $f_{\alpha}: X \rightarrow X_{\alpha}$ is $F$-supercontinuous. To show that the function $f$ is $F$-supercontinuous, it is sufficient to show that $f^{-1}(V)$ is $F$-open for each open set $V$ in the product space $\prod_{\alpha \in \Lambda} X_{\alpha}$. Since arbitrary unions and finite intersections of $F$-open sets is $F$-open, it suffices to prove that $f^{-1}(S)$ is $F$-open for every subbasic open set $S$ in the product space $\prod_{\alpha \in \Lambda} X_{\alpha}$. Let $V_{\beta} \times \prod_{\alpha \neq \beta} X_{\alpha}$ be a subbasic open set in $\prod_{\alpha \in \Lambda} X_{\alpha}$. Then $f^{-1}\left(V_{\beta} \times \prod_{\alpha \neq \beta} X_{\alpha}\right)=f^{-1}\left(p_{\beta}^{-1}\left(V_{\beta}\right)\right)=f_{\beta}^{-1}\left(V_{\beta}\right)$ is $F$-open in $X$. Hence $f$ is $F$-supercontinuous.

Theorem 4.11. For each $\alpha \in \Delta$, let $f_{\alpha}: X_{\alpha} \rightarrow Y_{\alpha}$ be a mapping and let $f: \prod X_{\alpha} \rightarrow \prod Y_{\alpha}$ be a mapping defined by $f\left(\left(x_{\alpha}\right)\right)=\left(f_{\alpha}\left(x_{\alpha}\right)\right)$ for each $\left(x_{\alpha}\right)$ in $\prod X_{\alpha}$. Then $f$ is $F$-supercontinuous if and only if $f_{\alpha}$ is $F$-supercontinuous for each $\alpha \in \Delta$.

Proof. Let $f: \prod X_{\alpha} \rightarrow \prod Y_{\alpha}$ be $F$-supercontinuous. Let $V_{\beta}$ be an open subset of $Y_{\beta}$. Then $V_{\beta} \times\left(\prod_{\alpha \neq \beta} Y_{\alpha}\right)$ is a subbasic open subset of the product space $\prod Y_{\alpha}$. Since $f$ is $F$-supercontinuous, $f^{-1}\left(V_{\beta} \times \prod_{\alpha \neq \beta} Y_{\alpha}\right)=f_{\beta}^{-1}\left(V_{\beta}\right) \times$ $\left(\prod_{\alpha \neq \beta} X_{\alpha}\right)$ is $F$-open in $\prod X_{\alpha}$. Consequently, $f_{\beta}^{-1}\left(V_{\beta}\right)$ is a $F$-open set in $X_{\beta}$ and hence $f_{\beta}$ is a $F$-supercontinuous.

Conversely, suppose that each $f_{\alpha}: X_{\alpha} \rightarrow Y_{\alpha}$ is $F$-supercontinuous. Let $V=V_{\beta} \times\left(\prod_{\alpha \neq \beta} Y_{\alpha}\right)$ be a subbasic open set in $\prod Y_{\alpha}$. Since each $f_{\alpha}$ is $F$ supercontinuous, and since $f^{-1}(V)=f^{-1}\left(V_{\beta} \times\left(\prod_{\alpha \neq \beta} Y_{\alpha}\right)\right)=f_{\beta}^{-1}\left(V_{\beta}\right) \times$ $\left(\prod_{\alpha \neq \beta} X_{\alpha}\right), f^{-1}(V)$ is $F$-open, and so $f$ is $F$-supercontinuous. 
Theorem 4.12. Let $f: X \rightarrow Y$ be a function and $g: X \rightarrow X \times Y$, defined by $g(x)=(x, f(x))$ for each $x \in X$, be the graph function. Then $g$ is $F$ supercontinuous if and only if $f$ is $F$-supercontinuous and $X$ is functionally regular.

Proof. To prove necessity, suppose that $g$ is $F$-supercontinuous. Then the composition $f=p_{y} \circ g$ is $F$-supercontinuous, where $p_{y}$ is the projection from $X \times Y$ onto $Y$. Let $U$ be any open set in $X$ and let $x \in U$. Then $U \times Y$ is an open set containing $g(x)$. Since $g$ is $F$-supercontinuous, there exists an $F$-open set $W_{x}$ containing $x$ such that $g\left(W_{x}\right) \subset U \times Y$. Thus $x \in W_{x} \subset U$ and since $U$ is a union of $F$-open sets, then it is $F$-open and so $X$ is functionally regular.

To prove sufficiency, let $x \in X$ and let $W$ be an open set containing $g(x)$. There exist open sets $U \subset X$ and $V \subset Y$ such that $(x, f(x)) \in U \times V \subset W$. Since $X$ is functionally regular, there exists an $F$-open set $G_{1}$ in $X$ containing $x$ such that $x \in G_{1} \subset U$. Since $f$ is $F$-supercontinuous, there exists an $F$-open set $G_{2}$ in $X$ containing $x$ such that $f\left(G_{2}\right) \subset V$. Let $G=G_{1} \cap G_{2}$. Then $G$ is an $F$-open set containing $x$ and $g(G) \subset U \times V \subset W$, which implies that $g$ is $F$-supercontinuous.

The following example shows that the hypothesis that ' $X$ is functionally regular' in Theorem 4.12 cannot be omitted.

Example 4.13. Let $X=Y=\{a, b, c, d\}$. Let the topology on $X$ be given by $\tau=\{\phi, X,\{a, b\},\{d\},\{a, b, d\}\}$ and let $Y$ be equipped with indiscrete topology. Let $f: X \rightarrow Y$ be the constant function which takes the value $b$. Then $f$ is $F$-supercontinuous but the graph function $g: X \rightarrow X \times Y$ is not $F$ supercontinuous.

Theorem 4.14. Let $f, g: X \rightarrow Y$ be F-supercontinuous functions from $X$ into a Hausdorff space $Y$. Then the equalizer $E=\{x \in X: f(x)=g(x)\}$ of the functions $f$ and $g$ is an $F$-closed set in $X$.

Proof. To show that $E$ is $F$-closed, we shall show that its complement $X \backslash E$ is an $F$-open subset of $X$. Let $x \in X \backslash E$. Then $f(x) \neq g(x)$. Since $Y$ is Hausdorff, there exist disjoint open sets $V$ and $W$ containing $f(x)$ and $g(x)$, respectively. Since $f$ and $g$ are $F$-supercontinuous, $f^{-1}(V)$ and $g^{-1}(W)$ are $F$-open sets containing $x$. Then $U=f^{-1}(V) \cap g^{-1}(W)$ is an $F$-open set containing $x$ which is contained in $X \backslash E$ and so $X \backslash E$ is $F$-open.

Corollary 4.15. Let $X$ be a Hausdorff space. Then the set of fixed points of every $F$-supercontinuous function $f: X \rightarrow X$ is an $F$-closed set.

Definition 4.16. A space $X$ is said to be $\boldsymbol{F}$-completely regular if for every $F$-closed set $A$ and a point $x$ outside $A$ there exists a continuous function $f: X \rightarrow[0,1]$ such that $f(x)=0$ and $f(A)=1$.

Theorem 4.17. Let $f: X \rightarrow Y$ be an F-supercontinuous function. If $X$ is $F$-completely regular, then $f$ is $z$-supercontinuous. 
Proof. Let $x \in X$ and let $V$ be an open set containing $f(x)$. Since $f$ is $F$ supercontinuous, there exists an $F$-open set $U$ containing $x$ such that $f(U) \subset$ $V$. Since $X$ is a $F$-completely regular space, there exists a continuous function $h: X \rightarrow[0,1]$ such that $h(x)=0$ and $h(X \backslash U)=1$. Then $h^{-1}[0,1)$ is a cozero set containing $x$ and contained in $U$ and so it is mapped into $V$ by $f$. This shows that $f$ is $z$-supercontinuous.

\section{Properties of the Graph of an $F$-Supercontinuous Function}

Let $f: X \rightarrow Y$ be an $F$-supercontinuous function. Since every $F$-supercontinuous function is continuous, the family $\left\{1_{x}, f\right\}$, where $1_{x}$ denotes the identity mapping on $X$, separates points and separates points from closed sets. Therefore, the mapping $g: X \rightarrow X \times Y$ defined by $g(x)=(x, f(x))$ is an embedding of $X$ into $X \times Y$. Thus $X$ is homeomorphic to its graph $G(f)=g(x)$ and so every topological property enjoyed by $X$ is also enjoyed by its graph $G(f)$.

The next two notions reflect upon the fact that how the graph $G(f)$ of an $F$-supercontinuous function $f: X \rightarrow Y$ is situated in the product space $X \times Y$.

Definition 5.1. Let $f: X \rightarrow Y$ be a function from a topological space $X$ into a topological space $Y$. The graph $G(f)$ of $f$ is said to be

(i) F-closed with respect to $X$ if for each $(x, y) \notin G(f)$, there exist open sets $U$ and $V$ containing $x$ and $y$, respectively such that $U$ is $F$-open and $(U \times V) \cap G(f)=\phi$.

(ii) $\mathbf{F}$-closed with respect to $\mathbf{X} \times \mathbf{Y}$ if for each $(x, y) \notin G(f)$, there exist $F$-open sets $U$ and $V$ containing $x$ and $y$, respectively such that $(U \times V) \cap$ $G(f)=\phi$.

Proposition 5.2. For a topological space $X$ the following are equivalent.

(a) $X$ is functionally Hausdorff.

(b) Every pair of distinct points in $X$ are contained in disjoint cozero sets.

(c) Every pair of distinct points in $X$ are contained in disjoint $F$-open sets.

Proof. The implication (a) $\Rightarrow(\mathrm{b}) \Rightarrow(\mathrm{c})$ are trivial. To prove (c) $\Rightarrow(\mathrm{a})$, let $x, y \in$ $X, x \neq y$ and let $U$ and $V$ be disjoint $F$-open sets containing $x$ and $y$, respectively. Let $A$ and $B$ be the zero sets in $X$ such that $x \in A \subset U$ and $y \in B \subset V$. Let $f, g$ be the real-valued functions defined on $X$ such that $Z(f)=A$ and $Z(g)=B$, where $Z(f)$ and $Z(g)$ denote the zero sets of $f$ and $g$, respectively. Let $h: X \rightarrow R$ be the function defined by $h(t)=f(t) /[f(t)+g(t)]$, for $t \in X$. Then $h$ is a continuous function defined on $X$ such that $h(x)=0$ and $h(y)=1$.

Theorem 5.3. If $f: X \rightarrow Y$ is $F$-supercontinuous and $Y$ is functionally Hausdorff, then $G(f)$, the graph of $f$ is $F$-closed with respect to $X \times Y$.

Proof. Let $x \in X$ and let $y \neq f(x)$. Since $Y$ is functionally Hausdorff, there exist disjoint $F$-open sets $V$ and $W$ containing $y$ and $f(x)$, respectively. By $F$-supercontinuity of $f$ there exists an $F$-open set $U$ containing $x$ such that 
$f(U) \subset W \subset Y \backslash \bar{V}$. Consequently, $U \times V$ contains no point of $G(f)$. Hence $G(f)$ is $F$-closed with respect to $X \times Y$.

The following result is immediate.

Proposition 5.4. If $f: X \rightarrow Y$ is $F$-supercontinuous and $Y$ is Hausdorff, then $G(f)$, the graph of $f$ is $F$-closed with respect to $X$.

\section{Topological properties and $F$-SUPercontinuity}

Theorem 6.1. Let $f: X \rightarrow Y$ be an $F$-supercontinuous open bijection. Then $X$ and $Y$ are homeomorphic functionally regular spaces.

Proof. Let $U$ be an open set in $X$ and let $x \in U$. Since $f$ is an open map, $f(U)$ is an open set containing $f(x)$. Since $f$ is $F$-supercontinuous, there exists an $F$-open set $G$ containing $x$ such that $f(G) \subset f(U)$. Now, $x \in f^{-1}(f(G)) \subset f^{-1}(f(U))$. Since $f$ is a bijection, $f^{-1}(f(G))=G$ and $f^{-1}(f(U))=U$. Thus $x \in G \subset U$. So $U$ being a union of $F$-open sets is $F$ open. Thus $X$ is a functionally regular space. Since $f$ is a homeomorphism and functional regularity is a topological property, $Y$ is functionally regular.

Theorem 6.2. Let $f: X \rightarrow Y$ be an F-supercontinuous injection into a $T_{0}$ space, then $X$ is a functionally Hausdorff space.

Proof. Let $x$ and $y$ be two distinct points in $X$. Then $f(x) \neq f(y)$. Since $Y$ is a $T_{0}$-space, there exists an open set $V$ containing one of the points $f(x)$ or $f(y)$ but not the other. To be precise, assume that $f(x) \in V$. Since $f$ is $F$-supercontinuous, $f^{-1}(V)$ is an $F$-open set containing $x$ but not $y$. So there exists a zero set $Z_{x}$ such that $x \in Z_{x} \subset f^{-1}(V)$. Let $\varphi: X \rightarrow[0,1]$ be the continuous function such that $Z(\varphi)=Z_{x}$ the zero set of $\varphi$. Then $\varphi(x)=0 \neq \varphi(y)$. Let $G$ and $H$ be disjoint open sets in [0,1] containing $\varphi(x)$ and $\varphi(y)$ respectively. It follows that $\varphi^{-1}(G)$ and $\varphi^{-1}(H)$ are disjoint cozero sets in $X$ containing $x$ and $y$, respectively. So $X$ is a functionally Hausdorff space.

Corollary 6.3. Let $f: X \rightarrow Y$ be a z-supercontinuous injection into a $T_{0}$ space, then $X$ is a functionally Hausdorff space.

Definition 6.4 ([13]). A space $X$ is said to be $\boldsymbol{F}$-compact if every F-open cover of $X$ has a finite subcover.

Theorem 6.5. If $f: X \rightarrow Y$ is an F-supercontinuous surjection from an $F$-compact space $X$ onto $Y$, then $Y$ is compact.

Proof. Let $\beta=\left\{V_{\alpha} \mid \alpha \in \Delta\right\}$ be an open cover of $Y$. In view of $F$-supercontinuity of $f,\left\{f^{-1}\left(V_{\alpha}\right): \alpha \in \Delta\right\}$ is an $F$-open cover of $X$. Since $X$ is $F$-compact, there exists a finite subset $\left\{\alpha_{1}, \ldots, \alpha_{n}\right\}$ of $\Delta$ such that $\bigcup_{i=1}^{n} f^{-1}\left(V_{\alpha_{i}}\right)=X$. Since $f$ is surjection, $\left\{V_{\alpha_{1}}, \ldots, V_{\alpha_{n}}\right\}$ is a finite subcover of $Y$. 
Definition 6.6 ([13]). A space $X$ is said to be weakly $\boldsymbol{F}$-normal if every pair of disjoint $F$-closed sets are contained in disjoint open sets.

Theorem 6.7 ([13]). Let $f: X \rightarrow Y$ be an F-supercontinuous closed surjection. If $X$ is a weakly $F$-normal space, then $Y$ is a normal space.

\section{F-Quotient Topology and F-Quotient SPACES}

Let $f: X \rightarrow Y$ be a surjection from a topological space $X$ onto a set $Y$. The quotient topology on $Y$ is the finest topology on $Y$, which makes $f$ continuous. Several variants of quotient topology have been defined in the literature (see $[8$, $10,11,15,22$, and 23]) which in general are weaker than quotient topology and coincide with the quotient topology if the domain is suitably augmented. For interrelations among these variants of quotient topology we refer the interested reader to [15]. In this section we introduce the notion of $F$-quotient topology which in general lies strictly between the quotient topology and the $z$-quotient topology [8].

We may recall that a set $U$ in a space $X$ is said to be $z$-open if it is expressible as a union of cozero sets in $X$.

Definition 7.1. Let $p: X \rightarrow Y$ be a surjection from a topological space $X$ onto a set $Y$.

(i) The collection $\tau$ of all subsets $A \subset Y$ such that $p^{-1}(A)$ is z-open in $X$ is a topology on $Y$ and is called $\mathbf{z}$-quotient topology [8] and the map $p$ is called the $\mathbf{z}$-quotient map.

(ii) The collection $\tau$ of all subsets $A \subset Y$ such that $p^{-1}(A)$ is F-open in $X$ is a topology on $Y$ and is called $\boldsymbol{F}$-quotient topology and the map $p$ is called the $\boldsymbol{F}$-quotient map.

Clearly, z-quotient topology $\subset F$-quotient topology $\subset$ quotient topology.

However, none of the above inclusions are reversible as is well exhibited by the following examples.

Example 7.2. Let $(X, \tau)$ be the space of Example 3.8. Then $X$ is a functionally regular space which is not a completely regular space. Let $Y=X$ and let $p$ denote the identity map defined on $X$. Then $p$ is an $F$-supercontinuous function which is not z-supercontinuous. The F-quotient topology on $Y$ is identical with $\tau$ while z-quotient topology is strictly coarser than $\tau$.

Example 7.3. Let $X$ be the space of all positive integers endowed with the prime integer topology $\sigma$ [24, Example 61, p. 82]. Then $X$ is a Hausdorff space which is not a functionally Hausdorff space and hence not a functionally regular space. Let $Y=X$ and let $p$ denote the identity map defined on $X$. Then the quotient topology on $Y$ is same as prime integer topology $\sigma$ but F-quotient topology on $Y$ is strictly coarser than $\sigma$.

Theorem 7.4. Let $p: X \rightarrow Y$ be a surjection from a topological space $X$ onto a topological space $(Y, \tau)$, where $\tau$ is the F-quotient topology on $Y$. Then $p$ 
is F-supercontinuous. Moreover, $\tau$ is the finest topology on $Y$ which makes $p: X \rightarrow Y, F$-supercontinuous.

Proof. $F$-supercontinuity of $p$ is an immediate consequence of the definition of $F$-quotient topology. Now let $\tau_{1}$ be a topology on $Y$ such that $p: X \rightarrow\left(Y, \tau_{1}\right)$ is $F$-supercontinuous. Let $G$ be a $\tau_{1}$ open set in $Y$. By $F$-supercontinuity of $p, p^{-1}(G)$ is $F$-open in $X$. Now by the definition of $F$-quotient topology, $G$ is $\tau$-open and hence $\tau_{1} \subset \tau$.

In contrast with the quotient space, the following result shows that a function out of an $F$-quotient space is continuous if and only if its composition with the $F$-quotient map is $F$-supercontinuous.

Theorem 7.5. Let $p: X \rightarrow Y$ be an F-quotient map. Then a function $g$ : $Y \rightarrow Z$ is continuous if and only if $g \circ p$ is F-supercontinuous.

Proof. Let $U$ be an open set in $Z$ and $g \circ p$ is $F$-supercontinuous, then $(g \circ$ $p)^{-1}(U)=p^{-1}\left(g^{-1}(U)\right)$, is $F$-open in $X$. Since $p$ is an $F$-quotient map, $g^{-1}(U)$ is open in $Y$. Hence, $g$ is continuous.

The converse is immediate.

\section{Change of Topology}

If the topology of domain of an $F$-supercontinuous function is changed in an appropriate way, then $f$ is simply a continuous function. For, let $(X, \tau)$ be a topological space, and let $\beta$ denote the collection of all $F$-open subsets of $(X, \tau)$. Since the intersection of two $F$-open sets is $F$-open, the collection $\beta$ is a base for a topology $\tau_{F}$ on $X$. Indeed $\beta=\tau_{F}$ and $\tau_{F} \subset \tau$. The space $(X, \tau)$ is functionally regular if and only if $\tau_{F}=\tau$.

Further, if $B_{z}$ denotes the collection of all cozero subsets of $(X, \tau)$, then $B_{z}$ is a base for a topology $\tau_{z}$ on $X$. It is immediate that $\tau_{z} \subset \tau_{F} \subset \tau$ and the space $X$ is completely regular if and only if $\tau=\tau_{z}$. Thus for a completely regular space $\tau_{z}=\tau_{F}=\tau$.

Throughout the section, the symbol $\tau_{F}$ will have the same meaning as in the above paragraph.

Remark 8.1. Any topological property which is invariant under continuous bijection will be transferred from $(X, \tau)$ to $\left(X, \tau_{F}\right)$. The list of such properties is fairly long. In particular, if $(X, \tau)$ is compact Lindelöf or countably compact, pseudocompact or quasicompact [5], D-compact or $D^{*}$-compact or $D_{\delta}$-compact [12], separable, connected or pathwise connected, then so is $\left(X, \tau_{F}\right)$.

Theorem 8.2. A function $f:(X, \tau) \rightarrow(Y, \Im)$ is $F$-supercontinuous if and only if $f:\left(X, \tau_{F}\right) \rightarrow(Y, \Im)$ is continuous.

Many of the results studied in preceding sections follow now from above theorem and the corresponding standard properties of continuous functions.

Theorem 8.3. Let $(X, \tau)$ be a topological space. Then the following statements are equivalent. 
(a) $(X, \tau)$ is functionally regular.

(b) Every continuous function from $(X, \tau)$ into a space $(Y, \Im)$ is $F$-supercontinuous.

Proof. (a) $\Rightarrow$ (b) is obvious.

(b) $\Rightarrow\left(\right.$ a): Take $(Y, \Im)=(X, \tau)$. Then the identity function $1_{x}$ on $X$ is continuous, and hence $F$-supercontinuous. Hence by Theorem 8.2, $1_{x}:\left(X, \tau_{F}\right) \rightarrow$ $(X, \tau)$ is continuous. Since $U \in \tau$ implies $1_{x}^{-1}(U)=U \in \tau_{F}$, therefore $\tau \subset \tau_{F}$. Thus it follows that $\tau=\tau_{F}$, and so $(X, \tau)$ is a functionally regular.

Definition 8.4 ([9]). A function $f: X \rightarrow Y$ from a topological space $X$ into a topological space $Y$ is said to be $\boldsymbol{F}$-continuous if for each $x \in X$ and each $F$-open set $U$ containing $f(x)$ there exists an open set $V$ containing $x$ such that $f(V) \subset U$.

Theorem 8.5. Let $f:(X, \tau) \rightarrow(Y, \Im)$ be a function. Then

(a) $f$ is $F$-continuous if and only if $f:(X, \tau) \rightarrow\left(Y, \Im_{F}\right)$ is continuous.

(b) $f$ is $F$-open if and only if $f:\left(X, \tau_{F}\right) \rightarrow(Y, \Im)$ is open.

In view of Theorems 8.2 and 8.3, Theorem 4.8 can be restated as follows. If $f:\left(X, \tau_{F}\right) \rightarrow(Y, \Im)$ is a continuous open surjection and $g:(Y, \Im) \rightarrow(Z, v)$ is a function, then $g$ is continuous if and only if gof is continuous.

Moreover, $F$-quotient topology on $Y$ determined by the surjection $f:(X, \tau) \rightarrow$ $Y$ in Section 7 coincides with usual quotient topology on $Y$ determined by $f:\left(X, \tau_{F}\right) \rightarrow Y$.

\section{REFERENCES}

[1] C. E. Aull, Notes on separation by continuous functions, Indag. Math. 31 (1969), 458461.

[2] C. E. Aull, Functionally regular spaces, Indag. Math. 38 (1976), 281-288.

[3] N. Bourbaki, Elements of General Topology Part I, Hermann, Addison-Wesley, 1966.

[4] H. Brandenburg, On spaces with $G_{\delta}$-basis, Arch. Math. 35 (1980), 544-547.

[5] Z. Froli'k Generalization of compact and Lindelöf spaces, Czechoslovak. Math. J. 13 (84) (1959), 172-217 (Russian).

[6] N.C. Heldermann, Developability and some new regularity axioms, Can. J. Math. 33, no. 3 (1981), 641-663.

[7] E. Hewitt, On two problems of Urysohn, Ann. of Math. 47, no. 3 (1946), 503-509.

[8] J. K. Kohli and R. Kumar, z-supercontinuous functions, Indian J. Pure Appl. Math. 33, no. 7 (2002), 1097-1108.

[9] J. K. Kohli, D. Singh, R. Kumar and J. Aggarwal, Between continuity and set connectedness, preprint.

[10] J. K. Kohli and D. Singh, D-supercontinuous functions, Indian J. Pure Appl. Math. 32, no. 2 (2001), 227-235.

[11] J. K. Kohli and D. Singh, $D_{\delta}$-supercontinuous functions, Indian J. Pure Appl. Math. 34, no. 7 (2003), 1089-1100.

[12] J. K. Kohli and D. Singh, Between compactness and quasicompactness, Acta Math. Hungar. 106, no. 4 (2005), 317-329.

[13] J. K. Kohli, D. Singh and J. Aggarwal, On certain weak variants of normality and factorizations of normality, preprint.

[14] J. K. Kohli, D. Singh and J. Aggarwal, R-supercontinuous functions, communicated.

[15] J. K. Kohli, D. Singh and R. Kumar, Some properties of strongly $\theta$-continuous functions, Bull. Cal. Math. Soc. 100 (2008), 185-196. 
[16] N. Levine, Strong continuity in topological spaces, Amer. Math. Monthly 67 (1960), 269.

[17] J. Mack, Countable paracompactness and weak normality properties, Trans. Amer. Math. Soc. 148 (1970), 265-272.

[18] B. M. Munshi and D. S. Bassan, Super-continuous mappings, Indian J. Pure Appl. Math. 13 (1982), 229-236.

[19] T. Noiri, On $\delta$-continuous functions, J. Korean Math. Soc. 16 (1980), 161-166.

[20] T. Noiri, Supercontinuity and some strong forms of continuity, Indian J. Pure. Appl. Math. 15, no. 3 (1984), 241-250.

[21] I. L. Reilly and M. K. Vamanamurthy, On super-continuous mappings, Indian J. Pure. Appl. Math. 14, no. 6 (1983), 767-772.

[22] D. Singh, $D^{*}$-supercontinuous functions, Bull. Cal. Math. Soc. 94, no. 2 (2002), 67-76.

[23] D. Singh, cl-supercontinuous functions, Applied General Topology 8, no. 2 (2007), 293300 .

[24] L. A. Steen and J. A. Seeback, Jr., Counter Examples in Topology, Springer Verlag, New York, 1978.

[25] W. T. Van Est and H. Freudenthal, Trennung durch stetige Funktionen in topologischen Raümen, Indagationes Math. 15 (1951), 359-368.

RECEIVED JunE 2008

ACCEPted JANUARY 2009

\section{J. K. KoHLI (jk_kohli@yahoo.com)}

Department of Mathematics, Hindu College, University of Delhi, Delhi 110007 , India.

D. Singh (dstopology@rediffmail.com)

Department of Mathematics, Sri Aurobindo College, University of Delhi-South Campus, Delhi 110 017, India.

JEETENDRA AGGARWAL (jitenaggarwal@gmail.com)

Department of Mathematics, University of Delhi, Delhi 110 007, India. 